\title{
Star cluster evolution in dark matter dominated galaxies
}

\author{
Anneke Praagman*,a,b,c Jarrod Hurley $^{\mathrm{b}}$, Chris Power $^{\mathrm{d}}$ \\ ${ }^{a}$ School of Physics and Astronomy, University of Nottingham, Nottingham, NG7 2RD, UK \\ ${ }^{b}$ Centre for Astrophysics and Supercomputing, Swinburne University of Technology, PO Box 218, VIC 3122, Australia \\ ${ }^{c}$ Kapteyn Astronomical Institute, University of Groningen, Postbus 800, NL-9700 AV Groningen, the Netherlands \\ ${ }^{d}$ Department of Physics 85 Astronomy, University of Leicester, University Road, Leicester LE1 7RH, UK
}

\begin{abstract}
We investigate the influence of the external tidal field of a dark matter halo on the dynamical evolution of star clusters using direct $N$-body simulations, where we assume that the halo is described by a Navarro, Frenk \& White mass profile which has an inner density cusp. We assess how varying the mass and concentration of the halo affects the rate at which the star cluster loses mass and we find that increasing halo mass and concentration drives enhanced mass loss rates and in principle shorter cluster disruption timescales. In addition, we examine disruption timescales in a three-component model of a galaxy (bulge, disk and dark matter halo) and find good agreement with results based on an empirical model of the Galactic potential if we assume a halo mass of $\sim 10^{12} \mathrm{M}_{\odot}$. In general, dark matter halos are expected to contribute 'significantly to the masses of galaxies and should not be ignored when modelling the evolution of star clusters. We extend our results to discuss how this can have a potentially profound effect on the disruption timescales of globular clusters, suggesting that we may underestimate the rate at which primordial globular clusters are disrupted.
\end{abstract}

Key words: globular clusters: general, galaxies: halos, dark matter, methods: $n$-body simulations PACS: 98.10.+z, 98.35.Gi

\section{Introduction}

Globular clusters are compact stellar systems, each containing of order $10^{6}$ stars, that orbit around the centres of galaxies out to large radii. Our Galaxy hosts around '200 globular clusters and observations show that almost 'all galaxies host these systems, with giant ellipticals having the largest (relative) population (Brodie and Strader, 2006). Stellar population studies have revealed that globular clusters have ages up to $\sim 13$ Gyrs (e.g. Hansen et al., 2002; Chabover and Krauss, 2002). This implies that they formed within $\sim 1$ billion years of the Big Bang and so they represent fossil records of the earliest epoch of galaxy formation, which has led to much interest in their potential as probes of the high redshift Universe (Brodie and Strader, 2006).

However, we have as yet no compelling theory for globular cluster formation and evolution within a cosmological framework and this has important consequences for the kind of questions we can address using globular clusters and the strength of conclusions that we can draw. One particularly important consequence concerns the disruption of primordial globular clusters and the relation of the high redshift globular cluster population to the population that we observe at the present day. All star clusters

\footnotetext{
* Corresponding author.

Email addresses: a.k.praagman@alumnus.rug.nl (Anneke Praagman), jhurley@astro.swin.edu.au (Jarrod Hurley), chris.power@astro.le.ac.uk (Chris Power)
}

lose mass over time and this depends on a number of factors including initial conditions (e.g. initial mass profile of the star cluster, initial mass function of the stars, initial binary fraction), internal processes within the cluster (e.g. mass segregation, stellar mass loss), the orbital parameters of the cluster and the form and nature of the external tidal field within which the cluster orbits. The globular cluster population that we observe around galaxies at the present day has survived a Hubble time, but it is interesting to ask whether or not this population is representative of the primordial globular cluster population at high redshift. If not, this would imply that many primordial globular clusters disrupted on relatively short timescales, and that the clusters that survived to the present day are in a sense atypical. This introduces certain caveats where the present day population is used to probe, say, the efficiency of star formation at high redshifts (Spitler and Forbes, 2008) and the epoch of cosmological reionisation (Moore et al., 2006).

In the standard picture of galaxy formation, galaxies are embedded in massive virialised structures or halos of dark matter (Springel et al., 2006). These dark matter halos assemble over time hierarchically, continuously growing via accretion of dark matter and merging with other halos from high redshift to the present day. The subset of globular clusters that have survived to the present day has evolved in a time-dependent and at times violently changing potential provided by its host galaxy and dark 
matter halo. Previous studies that have investigated star cluster mass loss (e.g. Giersz and Hegoie, 1997; Vesperini, 1998; Baumgardt, 2001; Hurley et al., 2001) - with models ranging in mass from open clusters to small globular clusters - have assumed simplistic models for the Galaxy's tidal field by treating the Galaxy as a central compact source or by using Oort constants that are empirically measured from stellar motions in the Solar neighbourhood (e.g. Binney and Tremaine, 1987). Mashchenko and Sills (2005) looked at the early evolution of globular clusters formed in dark matter halos but employed softened gravitational potentials for both the stars and dark matter. As such, the explicit presence 1 of a dark matter halo has been neglected to date in direct $N$-body models of star cluster evolution.

Our goal is to understand how the presence of a dark matter halo affects the internal characteristics of star clusters in general - and globular clusters in particular - and their disruption rates. For the former our interest is in signatures such as the ratio of the core to half-mass radii and the velocity dispersion, which may be compared to observations of star clusters. However, in this paper, the first in a series, we start by focusing on the rate of massloss from model star clusters and what this can tell us about the expected lifetimes and disruption of globular clusters. Our approach is to use a direct $N$-body code that treats the internal processes of a star cluster in as detailed a manner as possible. We include a spectrum of stellar masses and stellar evolution from the outset as the effect of these on star cluster evolution is relatively well understood (e.g. de La Fuente Marcos, 1996; Baumgardt and Makino, 2003; Hurley et al., 2004). In our treatment of the external potential and the orbits of the model clusters within this, we take a more cautious approach. We start with the simplest case, circular orbits in a variety of static potentials, as this facilitates comparison with previous $N$-body studies and provides the foundation for our study. In the future we will expand the models to include eccentric orbits and time-varying potentials. By necessity we also start with models more comparable in size to open clusters than globular clusters but this will also increase as we progress. In this way we build a consistent and realistic picture of star cluster evolution and survival.

We assess the influence of halo structure on mass loss rate using the functional form for the mass profile proposed by Navarro, Frenk, and White 1996, 1997, hereafter NFW). The NFW profile has been found to provide a good description of the spherically averaged dark matter distribution within halos in dynamical equilibrium in cosmological $N$-body simulations. The characteristic feature of the NFW profile is that it has a central density cusp, $\rho \propto r^{-1}$, so that the central density is divergent. We also construct a three-component model of our Galaxy consist-

\footnotetext{
${ }^{1}$ We use explicit because models of the Galactic potential based on Oort constants implicitly include the contribution of the Galaxy's dark matter halo.
}

ing of a bulge, disk and NFW halo and we compare the rate at which clusters lose mass in this model against the rate measured in the fiducial model of the Galactic tidal field, based on Oort constants.

The format of the paper is as follows. In the next section we describe the NFW profile in more detail, while in section 3 we describe the model we have adopted for our star cluster. In section 4 we present the main results of our study, first considering the evolution of an isolated cluster; then the evolution of the cluster in external galactic potentials; then evolution in the potential of a NFW halo; and finally evolution in our three-component model of a galactic potential, which consists of a bulge, disk and NFW halo. Finally we discuss the main findings and implications of our study in section 5 .

\section{The NFW Mass Profile}

Dark matter halos that form in cosmological simulations are relatively complex structures - they are generally aspherical (e.g. Bailin and Steinmetz, 2005) and asymmetric (e.g. Gao and White, 2006), they have no simple boundary (e.g. Prada et al., 2006), and they contain a wealth of small scale structure (Gao et al., 2004). Despite this complexity, however, it is conventional to identify a halo as a spherical overdense region, typically of order 100 times the mean density of the Universe. The mass enclosed within this spherical overdensity defines the virial mass of the halo,

$$
M_{\mathrm{vir}}=\frac{4 \pi}{3} \Delta_{\mathrm{vir}} \rho_{\mathrm{crit}} r_{\mathrm{vir}}^{3}
$$

Here $\rho_{\text {crit }}=3 H^{2} / 8 \pi G$ is the critical density of the Universe and $r_{\text {vir }}$ is the virial radius, which defines the radial extent of the halo. $\Delta_{\text {vir }}$, the virial overdensity criterion, corresponds to the mean overdensity at the time of virialisation in the spherical collapse model, the simplest analytic model of halo formation (cf. Eke et al., 1996). Depending on redshift and cosmological parameters, $\Delta_{\text {vir }}$ varies between $\sim 100$ and $\sim 200$.

The mass profiles of dark matter halos in dynamical equilibrium forming in cosmological $N$-body simulations can be relatively well described by the NFW profile,

$$
\rho(r)=\frac{\rho_{\mathrm{crit}} \delta_{\mathrm{c}}}{r / r_{\mathrm{s}}\left(1+r / r_{\mathrm{s}}\right)^{2}},
$$

where $\rho_{\text {crit }}$ is the critical density of the Universe, $r_{\mathrm{s}}$ is the scale radius and $\delta_{\mathrm{c}}$ is the characteristic overdensity. The characteristic overdensity $\delta_{\mathrm{c}}$ is itself a function of $r_{\mathrm{s}}$,

$$
\delta_{\mathrm{c}}=\frac{\Delta_{\mathrm{vir}}}{3} \frac{c^{3}}{\ln (1+c)-c /(1+c)},
$$

where $c=r_{\text {vir }} / r_{\mathrm{s}}$ is the concentration.

Because the scale radius $r_{\mathrm{s}}$ and the characteristic overdensity $\delta_{\mathrm{c}}$ are related, equation 2 can be rewritten in terms of a single parameter, the concentration $c=r_{\mathrm{vir}} / r_{\mathrm{s}}$, such 


\section{Model Description}

$1 \quad$ Isolated cluster

$2 \quad$ Standard Galactic tidal field

3 Two-component Galaxy (disk and bulge)

$4 \quad$ NFW halo

$5 \quad$ Full Galaxy (disk, bulge and NFW halo)

Table 1: Overview of the models used in the simulations in terms of the treatment of the external tidal field.

that for a fixed concentration, the local density depends only on the normalised radius $r / r_{\text {vir }}$. Cosmological simulations have shown that virial mass $M_{\text {vir }}$ and concentration $c$ are correlated, such that the concentration increases as the virial mass decreases (e.g Bullock et al., 2001; Eke et al., 2001; Neto et al., 2007). As a rule of thumb, a typical $M_{\mathrm{vir}}=10^{10}\left(10^{12}, 10^{14}\right) \mathrm{M}_{\odot}$ halo has a concentration $c \simeq 15$ $(10,5)$.

The mass enclosed within a radius $r$ is obtained from equation 2 ,

$$
M(<r)=4 \pi \rho_{\text {crit }} \delta_{\mathrm{c}} r_{\mathrm{s}}^{3} g(r)
$$

where

$$
g(r)=\ln \left(1+r / r_{\mathrm{s}}\right)-\frac{r / r_{\mathrm{s}}}{1+r / r_{\mathrm{s}}} .
$$

This results in a radial acceleration directed towards the centre of the halo of

$$
a(r)=-4 \pi G \rho_{\text {crit }} \delta_{\mathrm{c}} r_{\mathrm{s}} g(r)\left(r / r_{\mathrm{s}}\right)^{-2} .
$$

It follows that the gradient in the radial acceleration (and consequently the force) is given by

$$
\frac{\partial a}{\partial r}=-2 \frac{a(r)}{r}-4 \pi G \rho(r) .
$$

This provides a measure of the tidal force across the cluster and in the absence of an extended dark matter halo the tidal force would reduce to the familiar result for a point mass,

$$
\frac{\partial a}{\partial r}=-2 \frac{G M(r)}{r^{3}}
$$

\section{The cluster model}

To study the evolution of a star cluster we used the $N$-body code NBODY6 (see Aarseth, 2003, for a full description). It uses Hermite integration with individual time-steps and does not use softening in the force equation. Stellar evolution is included using the algorithms provided by Hurley et al. (2000) as described in Hurlev (2008). The code also allows for binary formation and evolution. There are also options to include an external Galactic potential with the simplest option being a standard Galactic potential at $8.5 \mathrm{kpc}$ based on the local Oort constants (hereafter the standard Galactic tidal field). In most respects NBODY6 is very similar to its sister code NBODY4 (Aarseth, 1999; Hurley et al., 2001) except that the latter interfaces with special-purpose GRAPE hardware (Makino et al., 2003) to speed up the calculation of the gravitational forces between the stars. NBODY6 operates on standard hardware and our simulations are performed on the Swinburne supercomputer 2 .

Within NBODY6 there is the possibility of building a multi-component galaxy consisting of bulge (point mass), disk (Mivamoto and Nagai, 1975) and logarithmic potential (Aarseth, 2003). To this we have added the possibility of replacing the logarithmic potential with a potential based on the NFW-profile. This three-component galactic potential thus consists of a bulge, disk and NFW halo. In all other respects the NBODY6 software is the same as the version publicly available for download at http://www.ast.cam.ac.uk/ sverre/web/pages/nbody.htm with input options and parameters set as for the defaults suggested in the included manual and described by Aarseth (2003).

All our models start with single stars only and these are distributed according to the Plummer (1911) density model, an $n=5$ polytrope. We assume the stars are in virial equilibrium when setting the initial positions and velocities and take the initial virial radius to be $3 \mathrm{pc}$ which sets the length scale of the simulation. It should also be noted that the Plummer profile formally extends to infinite radius so a cut-off at 10 times the half-mass radius is applied to deal with rare cases of large distance Aarseth, 2003). To save computing time we did not run full globular cluster models (i.e. $N=10^{5}-10^{6}$ ). Instead we focus on models of 1000 stars. For one galaxy scenario we also ran $N=16000$ simulations in order to investigate the scaling of the results with $N$. The initial masses of the stars are distributed according to a Salpeter initial mass function (IMF: $n(M) \propto M^{-2.3}$ ) with a minimum and maximum stellar mass $m_{\min }=0.3 \mathrm{M}_{\odot}$ and $m_{\max }=30 \mathrm{M}_{\odot}$ respectively. The average stellar mass is $1 \mathrm{M}_{\odot}$. In general our clusters are placed on circular orbits in the stellar disk (if present) at a radius of $8.5 \mathrm{kpc}$ from the Galactic centre. This facilitates comparison with previous studies. However, we do briefly explore the effect of orbital distance by placing some clusters on orbits at radii of 4.5, 17 and $34 \mathrm{kpc}$. An overview of our models used in terms of the treatment of the external tidal field is shown in Table 1 .

\section{Results}

\subsection{Star Cluster Evolution in Isolation}

Our first model (Model 1) is a cluster in isolation. Its dynamical evolution depends only on internal processes such as mass segregation and stellar evolution. The evolution of the total mass of such a cluster is shown in Figure

\footnotetext{
${ }^{2}$ see $\overline{\text { http://astronomy.swin.edu.au/supercomputing/ }}$
} 


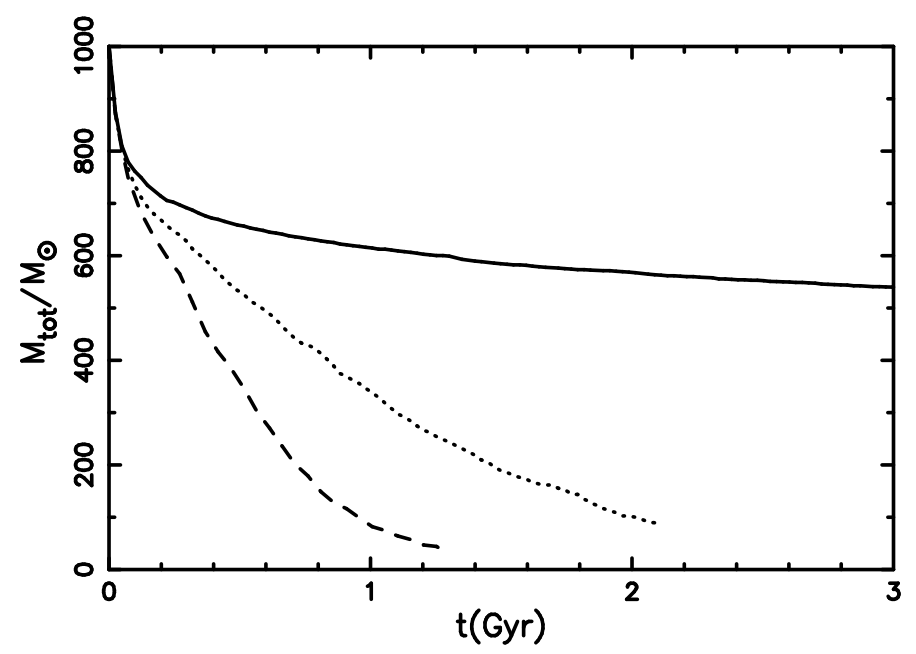

Figure 1: The evolution of the total mass of the cluster in isolation (Model 1, solid), in the standard Galactic tidal field (Model 2, dashed) and in the two-component galaxy (Model 3, dotted). All models start with $N=1000$.

1 by the heavy solid curve. In NBODY6 the cluster size is limited by a tidal radius or cut-off radius. For the case of an isolated cluster it is not meaningful to define a tidal radius and so we adopt a cut-off radius of 10 times the cluster scale length $r_{\mathrm{s}}$,

$$
r_{\mathrm{s}}=\frac{1}{2} \frac{G M_{\mathrm{tot}}^{2}}{\Phi},
$$

with $M_{\text {tot }}$ the total cluster mass and $\Phi$ the potential energy. Note that $r_{\mathrm{s}}$ is distinct from the NFW scale radius discussed in section 2. Stars with cluster-centric radii in excess of twice the cut-off radius are considered to have left the cluster and contribute to the mass loss. The initial cut-off radius is $23 \mathrm{pc}$, but this increases as the cluster expands to about $100 \mathrm{pc}$ after $3 \mathrm{Gyr}$.

Mass segregation causes the system to lose stars early on. Less massive stars gain kinetic energy through equipartition during encounters with more massive stars in the core and are more likely to escape. Stellar evolution leads to the loss of even more mass in the early stages. Massive stars evolve more rapidly than low mass stars and hence lose mass more quickly in the form of stellar winds. Neutron stars that form in a supernova will get a velocity kick and generally disappear from the system.

\subsection{The Standard Galactic Tidal Field Model}

Model 2 introduces the standard Galactic tidal field (SGTF) based on the local Oort constants $(A=14.4 \mathrm{~km} / \mathrm{s}$, $B=-12.0 \mathrm{~km} / \mathrm{s})$. These are derived empirically from the observed motions of stars in the Solar neighbourhood (Binney and Tremaine, 1987). As such they naturally account for the local effect of the total mass of the Galaxy (including its dark matter halo) and they strictly apply to orbits within the region of the Solar neighbourhood. From the local circular velocity we can infer that the enclosed mass at an orbit of $8.5 \mathrm{kpc}$ (the distance from the Sun to the Galactic centre) is $9 \times 10^{10} \mathrm{M}_{\odot}$. As the dashed curve in Figure 1 shows this has a profound effect on the mass loss of the cluster. There is an almost continuous mass loss leading to rapid disruption of the cluster: simulations are halted when the cluster has less than 25 stars left. Here we define the tidal radius as the King (1962) tidal radius,

$$
r_{\mathrm{t}}^{3}=\frac{1}{3} \frac{M_{\mathrm{C}}}{M_{\mathrm{G}}} R_{\mathrm{G}}^{3}
$$

with $M_{\mathrm{C}}$ and $M_{\mathrm{G}}$ the masses of the cluster and the galaxy respectively and $R_{\mathrm{G}}$ the distance between the galactic centre and the centre of the cluster. We set $R_{\mathrm{G}}=8.5 \mathrm{kpc}$ (the distance from our Sun to the Galactic centre). This gives a tidal radius of $\sim 16 \mathrm{pc}$, smaller than the cut-off radius used in Model 1. This has a bearing on what we compute as the mass loss rate, which will be lower in the case of the isolated cluster where the cut-off radius is higher. However, stellar evolution effects dominate in the isolated cluster so the general comparison holds.

\subsection{The Two-Component Model - Bulge 8 Disk}

Model 3 introduces the two-component model of the Galaxy, consisting of a bulge and disk. The disk is modelled as a Miyamoto-Nagai disk (Mivamoto and Nagai, 1975) and the bulge is a point mass at the centre of the galaxy. We use a mass of $5 \times 10^{10} \mathrm{M}_{\odot}$ for the disk, which has a radius of $40 \mathrm{kpc}$, and $1.5 \times 10^{10} \mathrm{M}_{\odot}$ for the bulge, as suggested by Xue et al. (2008) for the Milky Way.

We adopt a tidal radius of $16 \mathrm{pc}$, equal to the King radius (see Equation 10) of Model 2. This is kept fixed for all subsequent models at an orbital distance of $8.5 \mathrm{kpc}$, including the models where we add a dark matter halo. Thus we are effectively employing a tidal cut-off as described by Trenti et al. (2007).

The dotted line in Figure 1 shows the evolution of the cluster mass with Model 3 acting as the external tidal field. Model 3 does not produce a tidal field as strong as the SGTF of Model 2, but this is to be expected because the mass of the combined bulge and disk within $8.5 \mathrm{kpc}$ is smaller than the enclosed mass one infers for the SGTF of Model 2, which is $9 \times 10^{10} \mathrm{M}_{\odot}$. Recall that Model 2 is based on an empirical estimate of the Galactic tidal field and so implicitly includes the contribution of the Galaxy's dark matter halo, whereas Model 3 has no halo contribution.

The two-component model is attractive because we can fully determine how the Galactic potential is modelled and explore different sets of assumptions, but it neglects the presence of dark matter halo component to the potential which we expect to be important. In contrast, the SGTF model provides a realistic model of the Galactic potential in the Solar neighbourhood, being derived from empirical measurement, but the conditions under which it is applicable are restrictive. If the two-component model is to be made more realistic, then it must become a threecomponent model (a bulge, disk and halo), in which case 


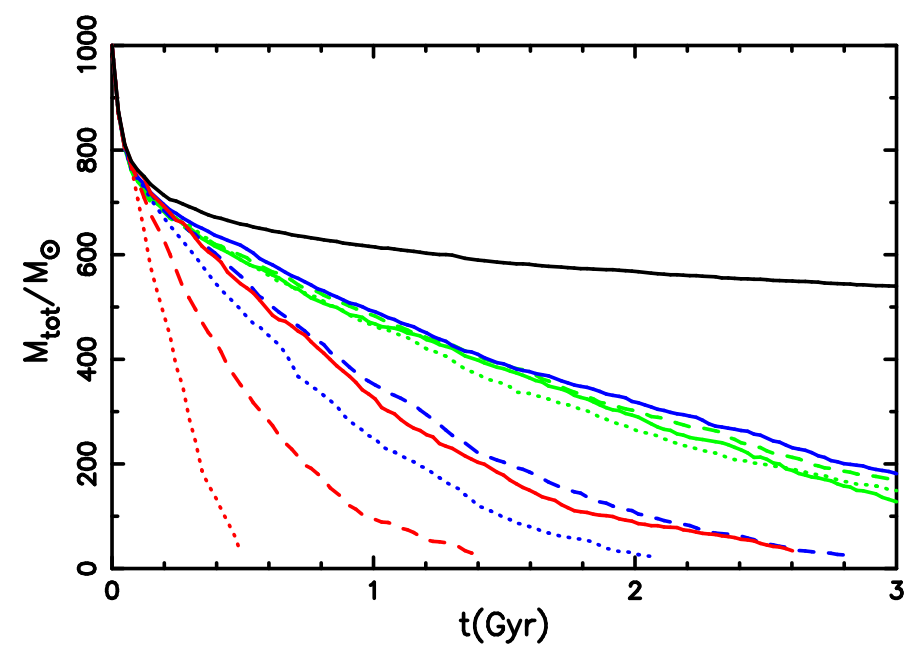

Figure 2: The total mass evolution of a cluster of 1000 stars in an NFW halo at an orbital distance of $8.5 \mathrm{kpc}$ from the halo centre (Model 4). We show here three different virial masses: $10^{9} \mathrm{M}_{\odot}$ (green), $1.5 \times 10^{12} \mathrm{M}_{\odot}$ (blue) and $10^{14} \mathrm{M}_{\odot}$ (red); each with three different concentrations: $c=5$ (solid), 10 (dashed) and 15 (dotted). The solid black line is Model 1, the cluster in isolation, and is drawn here as a reference.

we expect the mass loss rates for Models 2 and 3 to converge. In the next subsection we look at the influence of a single component model - that of a NFW dark matter halo on the mass loss rate - before introducing the threecomponent model in the final subsection.

\subsection{The NFW Halo}

We now consider a set of models in which our star clusters orbit in a NFW dark matter halo (Model 4). For now we ignore the stellar component of the galaxy because we want to investigate the influence of changing the NFW parameters of mass and concentration. We use a model grid of 6 different halo virial masses $\left(10^{9} \mathrm{M}_{\odot}, 10^{10} \mathrm{M}_{\odot}\right.$, $10^{11} \mathrm{M}_{\odot}, 1.5 \times 10^{12} \mathrm{M}_{\odot}, 10^{13} \mathrm{M}_{\odot}$ and $\left.10^{14} \mathrm{M}_{\odot}\right)$ and 3 concentrations (5, 10 and 15), thus 18 models in total. Here we define the virial mass as in equation 1 with an overdensity criterion of $\Delta_{\text {vir }}=200$. Each cluster follows a circular orbit at a distance of $8.5 \mathrm{kpc}$ from the centre of the halo. This is achieved by giving the cluster an initial circular velocity equal to the halo velocity at that distance,

$$
\left.V_{\mathrm{c}}=\sqrt{(G M}(<r) / r\right) .
$$

Our main set of simulations start with 1000 stars with a Salpeter IMF in the mass range $0.3-30.0 \mathrm{M}_{\odot}$ and total mass of $1000 \mathrm{M}_{\odot}$. Figure2 2 shows the evolution of the mass of the cluster. For clarity we only show 9 of the models representing the lowest mass, intermediate mass and highest mass halos. The mass loss is initially the same for all models since in this stage it is dominated by stellar evolution of the most massive stars. After about $100 \mathrm{Myr}$ clusters in the most massive halos keep losing mass at a faster pace

$\begin{array}{lllllrl}\begin{array}{l}\text { Mass } \\ \left(\mathrm{M}_{\odot}\right)\end{array} & c & \% r_{\mathrm{vir}} & \begin{array}{l}V_{c} \\ (\mathrm{~km} / \mathrm{s})\end{array} & \begin{array}{l}d V_{c} / d r \\ (\mathrm{~km} / \mathrm{s} / \mathrm{kpc})\end{array} & N & \begin{array}{r}t_{\mathrm{dis}} \\ (\mathrm{Myr})\end{array} \\ 1.0 \times 10^{9} & 5 & 52 & 12.46 & 0.68 & 1000 & 853 \\ 1.0 \times 10^{9} & 10 & 52 & 13.27 & 0.55 & 1000 & 916 \\ 1.0 \times 10^{9} & 15 & 52 & 13.66 & 0.49 & 1000 & 851 \\ & & & & & & \\ 1.0 \times 10^{10} & 5 & 24 & 17.77 & 1.27 & 1000 & 859 \\ 1.0 \times 10^{10} & 10 & 24 & 20.79 & 1.17 & 1000 & 921 \\ 1.0 \times 10^{10} & 15 & 24 & 22.42 & 1.08 & 1000 & 868 \\ & & & & & & \\ 1.0 \times 10^{11} & 5 & 11 & 22.68 & 2.00 & 1000 & 946 \\ 1.0 \times 10^{11} & 10 & 11 & 29.34 & 2.15 & 1000 & 826 \\ 1.0 \times 10^{11} & 15 & 11 & 33.51 & 2.15 & 1000 & 828 \\ & & & & & & \\ 1.5 \times 10^{12} & 5 & 4.6 & 26.75 & 2.75 & 1000 & 960 \\ 1.5 \times 10^{12} & 10 & 4.6 & 38.21 & 3.52 & 1000 & 614 \\ 1.5 \times 10^{12} & 15 & 4.6 & 46.69 & 3.94 & 1000 & 483 \\ & & & & & & \\ 1.5 \times 10^{12} & 5 & 4.6 & 26.75 & 2.75 & 16000 & 3749 \\ 1.5 \times 10^{12} & 10 & 4.6 & 38.21 & 3.52 & 16000 & 2746 \\ 1.5 \times 10^{12} & 15 & 4.6 & 46.69 & 3.94 & 16000 & 2152 \\ & & & & & & \\ 1.0 \times 10^{13} & 5 & 2.4 & 28.45 & 3.10 & 1000 & 825 \\ 1.0 \times 10^{13} & 10 & 2.4 & 42.60 & 4.34 & 1000 & 490 \\ 1.0 \times 10^{13} & 15 & 2.4 & 54.02 & 5.19 & 1000 & 324 \\ & & & & & & \\ 1.0 \times 10^{14} & 5 & 1.0 & 29.62 & 3.36 & 1000 & 587 \\ 1.0 \times 10^{14} & 10 & 1.0 & 45.90 & 5.03 & 1000 & 314 \\ 1.0 \times 10^{14} & 15 & 1.0 & 59.99 & 6.37 & 1000 & 190\end{array}$

Table 2: Disruption times - defined as the time after which the cluster has lost half of its initial mass - for clusters orbiting in NFW halos of different halo mass and concentration. We also show the percentage of the virial radius at which the cluster is orbiting at $8.5 \mathrm{kpc}$, the circular velocity and the gradient in the circular velocity at that radius, and the initial number of stars in the model.

than the clusters in the less massive halos. In these massive halos the effect of concentration is also clearly visible. Higher concentrations mean faster disruptions - more of the halo mass is concentrated within the cluster's orbit resulting in a greater tidal force on the cluster. The effects of concentration become negligible for the least massive halos, and it is also noteworthy that mass loss is very similar for the low- and intermediate-mass halos.

To quantify the disruption of a cluster we have defined the disruption time scale as the time it takes for the cluster to lose half of its mass. These timescales are tabulated in Table 2] Generally, clusters orbiting in more massive halos have shorter disruption times than those orbiting in less massive halos. In massive halos the concentration has a large effect on the disruption time while in the lower mass ranges the disruption is independent of mass and concentration. Variations in this regime are dominated by statistical effects.

We can understand why this might be by considering the tidal field across a cluster and determining how large it needs to be for the typical speeds of stars in the clus- 


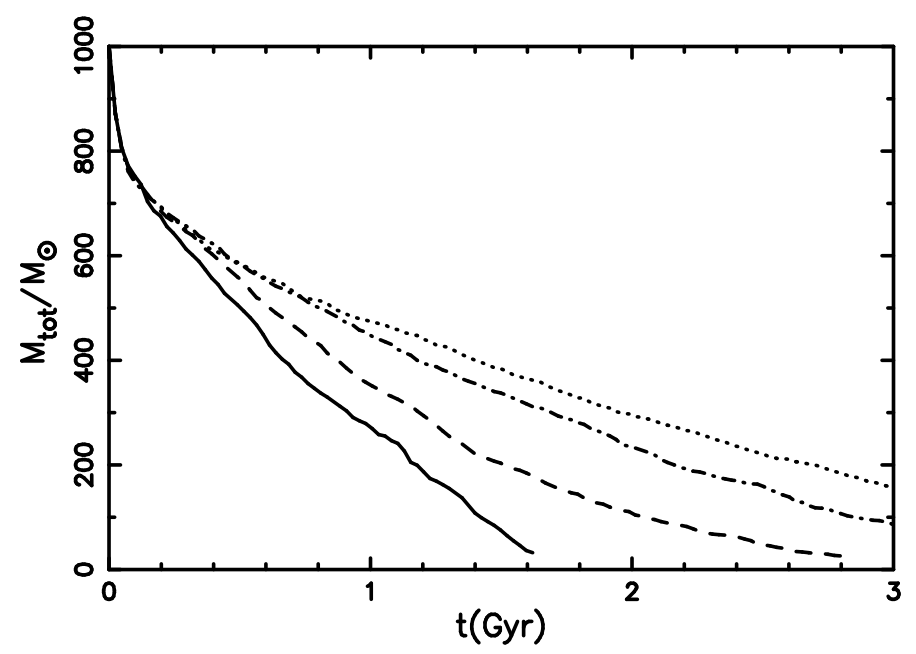

Figure 3: The mass loss for the standard NFW model $\left(M_{\mathrm{vir}}=\right.$ $\left.1.5 \times 10^{12} \mathrm{M}_{\odot}, c=10\right)$ at different orbital distances: $4.5 \mathrm{kpc}$ (solid), $8.5 \mathrm{kpc}$ (dashed), $17 \mathrm{kpc}$ (dashed-dotted) and $34 \mathrm{kpc}$ (dotted). All models start with $N=1000$.

ter to exceed the velocity dispersion. If $\sigma$ is the velocity dispersion of the cluster and $R_{\mathrm{c}}$ is its radius, then on a timescale $f \times R_{\mathrm{c}} / \sigma$ the net velocity of a star across the cluster is approximately $\partial a / \partial r \times 2 R_{\mathrm{c}} \times f \times R_{\mathrm{c}} / \sigma$. Here the factor $f$ will be greater than unity. If the test particle is to escape the cluster, $4 \times f \times\left(V_{\mathrm{c}} / R\right)^{2} \times R_{\mathrm{c}}^{2} / \sigma$ must be comparable to $\sigma$, where $V_{\mathrm{c}}$ is the halo circular velocity at halo-centric radius $R$. This reduces to the condition that

$$
\sigma \sim 2 \sqrt{f} V_{\mathrm{c}}\left(\frac{R_{\mathrm{c}}}{R}\right)
$$

For the star cluster mass we have considered, $\sigma \simeq 1 \mathrm{~km} / \mathrm{s}$ and $R_{\mathrm{c}} / R \simeq 0.001$, so $V_{\mathrm{c}} \sim 500 \mathrm{~km} / \mathrm{s}$ if $f=1$, or in other words in heavy halos clusters disrupt very efficiently. For less massive halos, we require $f \sim 100$, and so internal processes rather than the external tidal field will be important in driving the mass loss rate.

The model with virial halo mass of $1.5 \times 10^{12} \mathrm{M}_{\odot}$ and concentration $c=10$ resembles closest a model for our own Milky Way halo and from now on we will call it the standard NFW model. We ran $N=16000$ simulations for the models with this halo mass and the three different concentrations. The results are shown in Table 2 for comparison. The tidal radius for these larger models is $40 \mathrm{pc}$, as given by equation 10. We see the same general trend of decreasing disruption time with increasing concentration as seen for the $N=1000$ models. It has been shown previously by Baumgardt (2001) that the disruption time of a star cluster can be expected to scale by the initial half-mass relaxation timescale and an $N$ dependent factor of $(\log (0.11 N) / N)^{1 / 4}$ (see also Trenti et al., 2007). This is based on simulations of star clusters within a standard Galactic tidal field. The disruption timescales of our $N=16000$ models exceed those of their $N=1000$ coun-

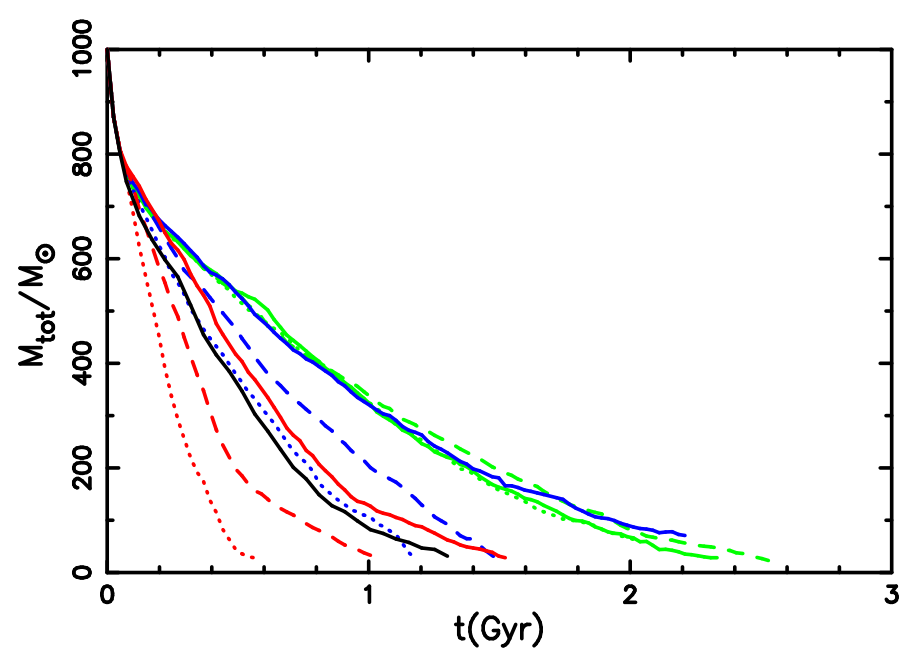

Figure 4: The total mass evolution for a cluster at an orbital distance of $8.5 \mathrm{kpc}$ within a full Galactic model (Model 5). Labels are as in Figure 2 except the solid black line now represents Model 2, with the tidal field based on the Oort constants. All models start with $N=1000$

terparts by a factor of $4-4.5$ while the half-mass relaxation timescale is a factor of two greater on average in the larger models. Thus we do not agree with the Baumgardt (2001) scaling relation by almost a factor of three. However, the use of both a tidal cut-off and a non-standard tidal field will certainly play a role and we will attempt to quantify this in future work after performing simulations for an expanded range of $N$.

For the standard NFW model we also looked at the effects of orbital distance. Figure 3 shows the mass evolution for this standard model at four different orbital distances. A smaller orbital radius leads to faster disruption. Note that the tidal radius remains fixed at $16 \mathrm{pc}$ for these models.

\subsection{The Three-Component Galaxy - Bulge, Disk \& Halo}

In Model 5 we added the two-component model Galaxy (Model 3) to the NFW models (Model 4), producing a three-component model consisting of bulge, disk and dark matter halo. Figure 4 shows the mass evolution for this model. The presence of the bulge and disk results in more rapid mass loss and consequently a shorter disruption time for the cluster. This is to be expected because of the addition of mass. The relative effect is greatest on the lowest mass halos because the size of the stellar component of the Galaxy is larger relative to the mass of their halo than is the case for larger halos. Comparing this to Model 2 (external field based on the local Oort constants) we see that it can be well reproduced by representing the galaxy as a disk, bulge and dark matter halo with a mass of $1.5 \times 10^{12} \mathrm{M}_{\odot}$ and concentration $\mathrm{c}=15$. The enclosed mass of the dark matter halo at an orbital distance of $8.5 \mathrm{kpc}$ is $9.4 \times 10^{10} \mathrm{M}_{\odot}$. This is of order $20 \%$ larger than what one infers from the Oort constants. All the models 
so far have assumed orbits in the disk. However we have looked at a model whose orbital is perpendicular to the plane of the disk; this resulted in an enhanced disruption rate because of "disk shocking": the cluster experiences a sudden change in the potential as it goes through the disk resulting in faster mass loss.

\section{Summary}

We have examined the importance the presence of a dark matter halo has on the rate at which a star cluster orbiting in its tidal field loses mass. For our halo model we adopted the NFW profile, which provides a good description of the mass profiles of dark matter halos forming in cosmological simulations. The NFW profile can be characterised by a single parameter, its concentration, which provides a measure of how rapidly the logarithmic slope changes with radius and consequently how rapidly the radial force changes with radius. As expected, star clusters orbiting at a fixed physical radius lose mass at a higher rate in more massive halos and in more concentrated halos.

We also investigated a three-component model of the Galactic tidal field, consisting of a stellar bulge and disk and a NFW halo and compared the mass loss rates of star clusters orbiting in this potential against mass loss rates of clusters orbiting in a standard Galactic tidal field (SGTF). The SGTF is based on empirical estimates of the Galactic tidal field in the Solar neighbourhood based on stellar motions, and as such provides a measure of total mass within the Solar radius, implicitly including our Galaxy's dark matter halo. We showed that star clusters orbiting in three-component models lose mass at a comparable rate to those orbiting in SGTF models if the halo mass is $1.5 \times 10^{12} \mathrm{M}_{\odot}$ and concentration $c=15$. These numbers are reasonable and consistent with what one would expect for Galactic-type dark matter halos in cosmological simulations. By building multi-component models of galactic potentials, one can explore many questions of cluster evolution that have not necessarily been possible before (such as the disruption timescales of clusters on eccentric orbits).

Our results are interesting because they highlight the importance of including an underlying dark matter halo in $N$-body simulations of long-term globular cluster evolution. Previous studies have neglected to do this, but as we have shown, the halo can have a profound effect on the lifetime of the star cluster. However, in the centre of the galaxy baryons will dominate the mass contribution. In future work we will also look at the effect of the halo mass on the internal properties of star clusters as they evolve. This is an important step in the spirit of the MODEST collaboration (Sills et al., 2003) to increase the realism of star cluster simulations. Of course, our calculation is idealised; we assume a static NFW halo - one that does not evolve over time - and our star clusters remain on a circular orbit at a fixed radius over a Hubble time. As we have noted in the introduction, we would expect a realistic halo to have had an active and at times violent mass assembly history, which should be an important additional driver of mass loss in our clusters. Furthermore, it is unclear what effect the assembly of the galaxy would have on the structure of the underlying dark matter halo. Nevertheless, our general conclusions hold and the effect of non-circular orbits and an evolving halo will be qualified as we continue our study.

Our results raise a number of interesting questions. The favoured cosmological model predicts that galaxies should live in cuspy dark matter halos (Springel et al., 2006). Previous studies of globular cluster evolution have been of clusters that evolve in non-cuspy potentials. Inferences have been drawn from these simulations of general processes such as core-collapse and mass-segregation and observations of globular clusters, which the simulations seem to do a good job of reproducing. We do not appear to need cuspy dark matter halos to produce these these trends in simulated globular clusters that are consistent with observational data, so does this imply that cuspy dark matter halos are not needed at all? This is not straightforward to answer, because it is not understood how the assembly of the galaxy has affected its dark matter halo, and whether this has effectively wiped out the cusp (as would appear necessary from rotation curve studies of dark matter dominated galaxies). However, if cusps are robust in the presence of the growing galaxy, then it may have implications for our understanding of globular cluster evolution.

More speculatively, the enhanced rate of disruption of clusters in cuspy halos has interesting implications for their use as probes of galaxy formation. Understanding how efficiently globular clusters form should provide us with insight into the efficiency of galaxy formation at high redshifts. The number of old globular clusters today could tell us how plentiful the sites of globular cluster formation were in the past, which in turn tells us about the efficiency with which gas cooled and formed molecular clouds. These sites may be regulated by the location (in the disks of gas-rich proto-galaxies, in mergers between gas-rich proto-galaxies) or the ambient radiation field (cosmological reionisation). If globular clusters are disrupted more efficiently in cuspy halos, then we could be missing a large fraction of globular clusters thus misinterpreting observations and drawing even more uncertain conclusions.

These are interesting questions and ones that we aim to revisit in coming papers.

\section{Acknowledgements}

CP is supported by the STFC rolling grant for theoretical astrophysics at University of Leicester.

\section{References}

Aarseth, S. J., Nov. 1999. From NBODY1 to NBODY6: The Growth of an Industry. PASP 111, 1333-1346. 
Aarseth, S. J., Nov. 2003. Gravitational N-Body Simulations. Gravitational N-Body Simulations, by Sverre J. Aarseth, pp. 430. ISBN 0521432723. Cambridge, UK: Cambridge University Press, November 2003.

Bailin, J., Steinmetz, M., Jul. 2005. Internal and External Alignment of the Shapes and Angular Momenta of $\Lambda$ CDM Halos. ApJ 627, $647-665$.

Baumgardt, H., Aug. 2001. Scaling of N-body calculations. MNRAS 325, 1323-1331.

Baumgardt, H., Makino, J., Mar. 2003. Dynamical evolution of star clusters in tidal fields. MNRAS 340, 227-246.

Binney, J., Tremaine, S., 1987. Galactic dynamics. Princeton, NJ, Princeton University Press, 1987, 747 p.

Brodie, J. P., Strader, J., Sep. 2006. Extragalactic Globular Clusters and Galaxy Formation. ARA\&A 44, 193-267.

Bullock, J. S., Kolatt, T. S., Sigad, Y., Somerville, R. S., Kravtsov, A. V., Klypin, A. A., Primack, J. R., Dekel, A., Mar. 2001. Profiles of dark haloes: evolution, scatter and environment. MNRAS 321, $559-575$.

Chaboyer, B., Krauss, L. M., Mar. 2002. Theoretical Uncertainties in the Subgiant Mass-Age Relation and the Absolute Age of $\omega$ Centauri. ApJ 567, L45-L48.

de La Fuente Marcos, R., Apr. 1996. The initial mass function and the dynamical evolution of open clusters. II. With mass loss. A\&A 308, 141-150.

Eke, V. R., Cole, S., Frenk, C. S., Navarro, J. F., Jul. 1996. Cluster correlation functions in N-body simulations. MNRAS 281, 703-+.

Eke, V. R., Navarro, J. F., Steinmetz, M., Jun. 2001. The Power Spectrum Dependence of Dark Matter Halo Concentrations. ApJ $554,114-125$.

Gao, L., White, S. D. M., Nov. 2006. Asymmetries in the inner regions of $\Lambda$ CDM haloes. MNRAS 373, 65-72.

Gao, L., White, S. D. M., Jenkins, A., Stoehr, F., Springel, V., Dec. 2004. The subhalo populations of $\Lambda$ CDM dark haloes. MNRAS 355, 819-834.

Giersz, M., Heggie, D. C., Apr. 1997. Statistics of N-body simulations - IV. Unequal masses with a tidal field. MNRAS 286, 709-731.

Hansen, B. M. S., Brewer, J., Fahlman, G. G., Gibson, B. K., Ibata, R., Limongi, M., Rich, R. M., Richer, H. B., Shara, M. M., Stetson, P. B., Aug. 2002. The White Dwarf Cooling Sequence of the Globular Cluster Messier 4. ApJ 574, L155-L158.

Hurley, J. R., 2008. N-Body Stellar Evolution. In: Aarseth, S. J., Tout, C. A., Mardling, R. A. (Eds.), Lecture Notes in Physics, Berlin Springer Verlag. Vol. 760 of Lecture Notes in Physics, Berlin Springer Verlag. pp. 283-+.

Hurley, J. R., Pols, O. R., Tout, C. A., Jul. 2000. Comprehensive analytic formulae for stellar evolution as a function of mass and metallicity. MNRAS 315, 543-569.

Hurley, J. R., Tout, C. A., Aarseth, S. J., Pols, O. R., May 2001. Direct N-body modelling of stellar populations: blue stragglers in M67. MNRAS 323, 630-650.

Hurley, J. R., Tout, C. A., Aarseth, S. J., Pols, O. R., Dec. 2004. Metallicity effects on open cluster dynamics. MNRAS 355, 12071216.

King, I., Oct. 1962. The structure of star clusters. I. an empirical density law. AJ 67, 471-+

Makino, J., Fukushige, T., Koga, M., Namura, K., Dec. 2003. GRAPE-6: Massively-Parallel Special-Purpose Computer for Astrophysical Particle Simulations. PASJ 55, 1163-1187.

Mashchenko, S., Sills, A., Jan. 2005. Globular Clusters with Dark Matter Halos. II. Evolution in a Tidal Field. ApJ 619, 258-269.

Miyamoto, M., Nagai, R., 1975. Three-dimensional models for the distribution of mass in galaxies. PASJ 27, 533-543.

Moore, B., Diemand, J., Madau, P., Zemp, M., Stadel, J., May 2006. Globular clusters, satellite galaxies and stellar haloes from early dark matter peaks. MNRAS 368, 563-570.

Navarro, J. F., Frenk, C. S., White, S. D. M., May 1996. The Structure of Cold Dark Matter Halos. ApJ 462, 563-+.

Navarro, J. F., Frenk, C. S., White, S. D. M., Dec. 1997. A Universal Density Profile from Hierarchical Clustering. ApJ 490, 493-+.

Neto, A. F., Gao, L., Bett, P., Cole, S., Navarro, J. F., Frenk, C. S.,
White, S. D. M., Springel, V., Jenkins, A., Nov. 2007. The statistics of $\Lambda$ CDM halo concentrations. MNRAS 381, 1450-1462.

Plummer, H. C., Mar. 1911. On the problem of distribution in globular star clusters. MNRAS 71, 460-470.

Prada, F., Klypin, A. A., Simonneau, E., Betancort-Rijo, J., Patiri, S., Gottlöber, S., Sanchez-Conde, M. A., Jul. 2006. How Far Do They Go? The Outer Structure of Galactic Dark Matter Halos. ApJ 645, 1001-1011.

Sills, A., Deiters, S., Eggleton, P., Freitag, M., Giersz, M., Heggie, D., Hurley, J., Hut, P., Ivanova, N., Klessen, R. S., Kroupa, P., Lombardi, Jr., J. C., McMillan, S., Portegies Zwart, S., Zinnecker, H., Aug. 2003. MODEST-2: a summary. New Astronomy 8, 605628

Spitler, L. R., Forbes, D. A., Nov. 2008. A new method for estimating dark matter halo masses using globular cluster systems. MNRAS, $\mathrm{L} 132+$.

Springel, V., Frenk, C. S., White, S. D. M., Apr. 2006. The largescale structure of the Universe. Nature 440, 1137-1144.

Trenti, M., Heggie, D. C., Hut, P., Jan. 2007. Star clusters with primordial binaries - II. Dynamical evolution of models in a tidal field. MNRAS 374, 344-356.

Vesperini, E., Oct. 1998. Evolution of the mass function of the Galactic globular cluster system. MNRAS 299, 1019-1039.

Xue, X. ., Rix, H. ., Zhao, G., Re Fiorentin, P., Naab, T., Steinmetz, M., van den Bosch, F. C., Beers, T. C., Lee, Y. S., Bell, E. F., Rockosi, C., Yanny, B., Newberg, H., Wilhelm, R., Kang, X., Smith, M. C., Schneider, D. P., Jan. 2008. The Milky Way's Circular Velocity Curve to $60 \mathrm{kpc}$ and an Estimate of the Dark Matter Halo Mass from Kinematics of 2400 SDSS Blue Horizontal Branch Stars. ArXiv e-prints 801 